\title{
XVIII' SIECLLE
}

\section{ACADEMIES ET SALONS}

\section{L'ENCYCLOPEDIE DE DIDEROT ET D'ALEMBERT}

M. Benr. - Nous arrivons à notre quatrième étape.

Avant-hicr, avec Bacon, nous avions vu un grand initiateur plutôt qu'un réalisateur : il avait ouvert à la science de vastes horizons spéculatifs, mais il n'a pas mis, si j'ose dire une peu familièrement, la main à la pâte. Hier, nous avons vu deux grands esprits qui, eux, ont mis la main à la pâte : l'un, Gassendi, avec quelques hésitations, quelques restes encore de la tradition du Moyen âge; Descartes, lui, avec force, avec audace, avec non seulement un pressentiment de la synthèse, mais niême une réalisation de la synthèse spéculative, et aussi un pressentiment très net de ce que la science au point de vue pratique, technique, pourrait donner.

Nous arrivons aujourd'hui à ce Xvili siècle qu'on appelle aussi le siècle des lumières, qu'en Allemagne on appelait l'Aufklärung. Et nous allons voir ce qui a été fait, non sans difficulté, sans péril par ceux qui entreprenaient la grande œuvre de science, tout ce qui a été fait dans le sens de la synthèse.

Nous entendrons parler d'abord des académies et des salons par Mlle Delorme qui, tout à l'heure, modestement voulait rester à sa place de secrétaire générale, mais que j'ai voulu voir à l'honneur dans le fauteuil du conférencier. Mlle Delorme rend de très grands services au Centre de Synthèse et à l'Institut international de Philosophie; et de plus, elle trouve le temps de mener des travaux personnels, qui 
sont en rapport avec ce qu'évoque cette salle même. En vous retournant vers le fond de la salle, vous pourrez voir un portrait de Fontenelle, qui a été un habitué de Mme de Lambert dans ce salon, et que nous considérons comme un des précurseurs de l'Encyclopédie. Mlle Delorme s'occupe de Fontenelle, et de toute cette époque; elle est tout à fait qualifiée pour nous parler des académies et des salons, de ce qui a précédé et préparé l'Encyclopédie.

Je donne la parole à Mlle Delorme.

Mlle Delonms. - Je remercie beaucoup M. Berr des paroles affectueuses qu'il vient de prononcer à mon égard. Je me trouve très émue, certes, de parler dans ce salon, devant ce tableau qui représente Fontenelle, de cette époque où ces grands hommes, ces grands académiciens, de la fin du siècle de Louis XIV au début du règne de Louis XV, ont si souvent tenu les propos les plus charmants, les plus agréables à entendre. Mais précisément, pour vous laisser un peu du charme du $x^{\prime} I^{\circ}$ siècle qu'évoque cette salle, je m'effacerai très souvent et je laisserai parler les textes. J'ai apporté beaucoup de volumes : je citerai beaucoup de textes. Et je commencerai par Fontenelle, évidemment, puisqu'il nous a donné lui-même une histoire des académies dans sa préface à l'Histoire de l'Académie royale des Sciences de 1666 à 1699 , tout au moins une histoire de l'Académie des Sciences. Cette préface date de $\mathbf{1 7 3 3}$. On avait alors décidé de combler la lacune qui se trouvait dans la collection complète de l'Histoire de l'Académie royale des Sciences: Fontenelle l'avait publiée en français depuis 1699. Mais pour la période précédente, depuis 1666, elle avait été écrite en latin par le secrétaire de l'Académie, Jean-Baptiste Duhamel. On préféra que toute l'Histoire fût en français et Fontenelle la rédigea pour les années 1666 jusqu'à 1679 , comme en témoigne l'avertissement de l'Histoire de l'Académie royale des Sciences depuis son établissement en 1666 jusqu'à 1686.

Dans cette préface de 1733 , Fontenelle nous rappelle ce qu'était le monde scientifique avant la création de l'Ácadémie en 1666; et je crois qu'il est nécessaire, pour moi aussi, de vous rappeler ce qu'était ce monde scientifique du xvi ${ }^{\bullet}$ sieccle avant d'aborder le xvir".

Nous retournons en arrière, au temps de Gassendi, de Descartes, à ce temps évoqué hier par M. Rochot, et peut-être même à l'esprit du $x v I^{*}$ siècle dont il a été question dans la 
conférence de $M$. Schuhl. M. Le Lionnais rappelait en effet hier, que le $\mathrm{x} v \mathrm{II}^{\bullet}$ sic̀cle était en relation étroite avec le $\mathrm{xvI}^{\bullet}$ au point de vue du désir de collectionner, de l'avidité du savoir. Ces sentiments se manifestent très nettement dans toutes ces socićtés savantes, à toutes ces conférences chez les amateurs savants qu'on rencontre à travers tout le milieu du xvit* siècle, et même un peu avant, depuis Mersenne chez qui l'on se réunissait avec Pascal.

Je cite Fontenelle : "Il y a déjà plus de cinquante ans que ceux qui étaient à Paris se voyaient chez le P. Mersenne, qui, étant ami des plus habiles gens de l'Europe, se faisait un plaisir d'être le lieu de leur commerce. MM. Gassendi, Descartes, Hobbes, Roberval, les deux Pascal père et fils, Blondel et quelques autres, s'assembloient chez lui. Il leur proposoit des Problèmes de Mathématique ou les prioit de faire quelques expériences par rapport à de certaines vues, et jamais. on n'avoit cultivé avec plus de soin les Sciences qui naissent de l'union de la Géométrie et de la Physique.

"Il se fit des assemblées plus régulières chez M. de Montmor, maître des Requêtes, et ensuite chez M. Thévenot '.

Jajouterai à ce que dit Fontenelle que d'autres réunions scientifiques, que les historiens ont considérées avec beaucoup trop de négligence, ont eu une très grande importance à l'époque. Ce sont les "Conférences ou assemblées de gens doctes et curicux des sciences et des arts, qui se tenaicnt tous les lundis de 2 à 4 heures, et où tous étaient reçus à donner leurs avis et à écouter ceux d'autrui sur la matière proposée $\triangleright$. Ces conférences avaient lieu au Bureau d'adresses de Théophraste Renaudot. Théophraste Renaudot a été l'initiateur de quantités de choses: depuis de nombreuses gazcttes jusqu'à un hôtel des ventes, un mont-de-pićté, des petites annonces, des consultations charitables pour les malades, un laboratoire de chimie, etc... Mais, ses conférences et assemblécs de gens doctes et curieux des sciences ont eu pendant plusieurs années un succès très grand. Du 22 août 1633 au $1^{\mathrm{er}}$ septembre 1642 , il y eut 355 conférences. Elles ont èté publiées sous le nom de * Première, deuxième... Centurie des questions traitées des conférences du Bureau d'adresses. ,

Ces questions n'avaient certainement pas un intérêt scien-

1. Histoire de l'Académie royale des Sciences, depuis son établissement en 1666 jusqu'en 1686. Paris, 1733, t. I, p. 3. 
tifique très grand; mais l'empressement manifesté à suivre ces réunions montre l'attrait très vif que tout ce monde du xvir siècle éprouvait pour ce problème : connaître le monde! On voit là une préoccupation tout à fait dans l'esprit du xvi' siècle; mais c'est déjà aussi la preuve du désir de s'assembler pour discuter sur un sujet mis à l'étude, en réunions qui sont la pré-formation des académies futures.

- Cette académie était ouverte à tout bon esprit qui y venait conférer en public de toutes les matières de physique, morale, et autres disciplines, et laquelle était une des plus belles et utiles institutions qu'eût faites Renaudot au jugement même de plusieurs de ses aînés. \C'est un texte du temps qui dit cela.

L'on trouve quantité de renseignements intéressants sur ces premières conférences dans un ouvrage en anglais de Harcourt Brown qui s'intitule Scientific Organizations in Seventeenth Century France (1620-1680) ${ }^{2}$. C'est un livre très précieux pour étudier ce début des organisations scientifiques et des académies scientifiques au xvı ${ }^{\circ}$ siècle.

Si j'ai voulu insister sur ce point, c'est qu'il est moins connu que le reste et que Fontenelle n'en parle pas. Il décrit l'Académie de $\mathbf{M}$. de Montmor, mais tout le monde la connaît. Elle avait même un règlement; elle étaił vraiment organisće; elle n'était pas sous le contrôle des pouvoirs officiels; mais les auditeurs devaient suivre certaines règles, qu'ils n'ont d'ailleurs pas toujours observées. C'est une des raisons pour lesquelles cette académie n'a pas été au fond vraiment reconnue par le roi. Bien que les règlements aient prescrit que le but de ces conférences ne doive pas être le vain exercice de l'esprit sur des subtilités inutiles », en fait, bien souvent ces conférences tournaient aux petits discours; l'on parlait beaucoup plus qu'on ne faisait des expériences. C'est là une des très grandes critiques qu'on pouvait faire: il n'y avait pas suffisamment d'expériences. On s'en tenait à des théories, à des subtilités, à des rêveries métaphysiques.

Les véritables savants comme Rohault, Auzout, Roberval, aussi, mais surtout Rohault et Auzout, se rendaient compte de la nécessité d'avoir beaucoup d'instruments et des laboratoires; mais cela demandait beaucoup d'argent. Pour faire de la recherche scientifique, il faut des crédits; et, à qui s'adresser pour en avoir? Au pouvoir central, à l'Etat; or

2. Baltimore, 1934. 
l'Etat à I'époque, c'est Louis XIV. Aussi Perrault, membre de ces sociétés, écrit-il à Colbert, en 1666, pour lui expliquer que les savants ont besoin d'aide : ils ont besoin de télescopes, et ce serait vraiment une excellente chose pour le prestige du roi d'être à la tête d'une académie qui, en même temps qu'elle servirait les sciences, servirait également le prestige royal. Mais, avant d'assister à la naissance de l'Académic royale des Sciences, revenons quelques années auparavant.

Outre les conférences tenues chez $M$. de Montmor, maitre des Requètes, où se réunissent Gassendi, Boulliau, Chapelain, Roberval, Petit, Rohault, Auzout, où Huyghens fait une communication, conférences qui se continuent chez Melchisédec. Thívenot en 1664-1665 et où fréquentent également le géomitre Frémile, le Danois Sténon, labbé Charles, collectionneur d'optiques, assemblée dont Auzout voudrait faire une «Compagnie des Sciences et des Arts », il nous faut citer encore la «Conférence de l'abbé d'Aubignac $\gg$ où l'on défend la physique moderne de Gassendi contre Aristote et Descartes. En 1656, il s'y manifeste le désir d'ćtablir une seconde académie officiclle, à côté de l'Académie française, projet qui n'a pas de suite immédiate, mais qui prépare l'opinion à l’idće d'autres académies.

Fontenelle suggère que ces assemblées de Paris * ont donné occasion à la naissance de plusieurs académies dans le reste de l'Europe... Il est toujours certain que les gentilshommes anglois qui ont jeté les premiers fondements de la Société royale de Londres, avoient voyagé en France, et s'étoient trouvés chez MM. de Montmor et Thévenot». Il est, en effet, tout a fait exact que les ctrangers qui venaient à Paris, avaient beaucoup de plaisir à être introduits dans les conférences soit de Montmor, soit de Thévenot. Ils repartaient, en Angleterre, en particulier, avec le grand désir d'entretenir une correspondance assidue avec ces savants. Mais, de là à conclure qu'il faut voir, ici, comme le dit Fontenelle, l'origine de la Société royale de Londres, M. Brown montre très pertinemment que ce n'est pas tout à fait exact.

Fontenelle écrit : «Quand ils furent de retour en Angleterre. ils retournèrent à Oxford et continuèrent les exercices auxquels ils s'étaient accoutumés en France avant leur retour en Angleterre. $\gg$ Ce n'est pas du tout comme cela que les choses se sont passées. Les savants anglais Boyle, Jean Walis, Thomas Willis se réunissaient à Oxford bien avant qu'Oldenbourg ne soit venu à Paris. Ils se réunissaient chez 
Wilkins, docteur du Collège de Wadham; et aussi, à la mêne époque, il y avait des réunions bi-hebdomadaires au Collège de Gresham. Ce sont ces réunions-là qui ont constitué la Société royale de Londres, laquelle a obtenu des lettres patentes de Charles II, en 1660.

D'après l'histoire de Thomas Sprat, parue au milieu du xvir' siècle, le but de la Société royale de Londres était de - recueillir de fidèles mémoires de tous les ouvrages de la nature et de l'art, à la connaissance desquels on peut parvenir, rétablir les vérités qui avaient paru négligées, en séparer les préjugés et les abus en les faisant connaitre et en les réfutant ?.

La naissance de la Société royale de Londres nous apparaft comme tout à fait différente de celle de l'Académie royale des Sciences : cela s'explique par les différences de caractère entre les nations et les esprits anglais et français.

La Société royale de Londres est une société particulière qui devient Socićté royale, alors que l'Acadénie royale des Sciences n'est autre que les assemblées réunies chez les particuliers. D'autre part, le règlement est très différent de celui qui sera donné plus tard à l'Académie royale des Sciences : les membres de la Société royale de Londres paient une cotisation; ils ne sont pas choisis par le roi; s'ils sont jugés dignes par leurs collègues d'entrer dans la Société, il leur suffit de payer un droit d'entrée, puis une cotisation annuelle; le nombre des membres est illimité. Il y a là des différences très grandes avec l'Académie royale des Sciences, que Voltaire notera plus tard très bien dans Les Lettres philosophiques en donnant tort aux Anglais (ce qui ne lui arrive pas souvent). Il dit que ce qui manque à la Société royale de Londres, ce sont des règles bien établies et des récompenses, et que ce qui précisément donne une certaine force à l'Académie royale des Sciences c'est qu'elle a un bon règlement qu'on ne doit pas transgresser; d'autre part, les hommes agissant très souvent dans l'espoir d'être récompensés, les prix excitent le travail scientifique ${ }^{3}$.

Nous voici arrivés en 1666 . Toutes les sociétés locales, particulières, continuent plus ou moins d'exister; mais Colbert ne veut donner le sceau royal à aucune d'entre elles, à la différence de Londres. Et il va créer, sur les conseils d'Auzout et de Perrault, une société qui ne dépendra que de lui et du

3. Voutainz, Lettres philosophiques, XXIV. 
roi. Et il va choisir différents membres dans diverses sociétés pour constituer la première société scientifique officielle de France, l'Académie des Sciences.

Fontenelle dans cette préface que j'ai déjà citée, où il relate les origines de l'Académie royale Sciences nous dit qu'au début on avait pensée à une académie unique pour les Belles-Lettres et pour les Sciences; mais on a vite constaté qu'un tel projet n'était pas viable et qu'il valait mieux faire une Académie des Sciences tout à fait autonome.

Aussi la «Petite Académie \& qui s'occupait des médailles, de l'examen des sujets de tapisseries, des projets pour Versailles, de l'histoire des grands faits du règne de Louis XIV (il n'était pas encore très long), continua d'exister, et plus tard, en 1709 , elle recevra un règlement, des lettres patentes et elle deviendra l'Académie des Inscriptions et des Médailles; ce n'est qu'ensuite, en 1716, qu'elle prit le titre d'Académie des Inscriptions et Belles-Lettres.

L'Académie des Sciences est donc restée indépendante de toute autre occupation : clle s'est consacrée exclusivement aux Sciences, différant encore en cela de la Société de Londres qui s'occupait arssi bien de morale, de théologie, d'histoire, de belles-lettres, que de sciences. Elle possédait cette même originalité vis-à-vis des innombrables académics qui florissaient en Italie. Toutes les grandes villes d'Italie avaient, en effet, leur académie; les plus célèbres sont évidemment celle de Florence, l'Académie del Cimento, et celle de Rome, l'Académia dei Lincei qui n'a pas un caractère purement scientifique. Les autres petites académies d'Italie sont piesque toutes des académies des Belles-Lettres et non des académies scientifiques, à l'exception cependant de l'Institut de Bologne qui sera créé bien plus tard, en 1712, par Marsigli, où l'on réunira l'Académie des Philosophes inquiets qui existait auparavant : ces philosophes inquiets étaient destinés a travailler sans relâche à la perfection * des arts et des sciences $\downarrow$.

Ces remarques nous ont éloignés un peu de l'Académie royale des Sciences. Reprenons son histoire avec Fontenelle. C'est en juin 1666, qu'eurent lieu les premiers exercices académiques dans la Bibliothèque de $M$. Colbert où s'assemblaient \& les Mathématiciens en nombre de six ou sept, MM. Carcavy, Huyghens, Roberval, Frémile, Auzout, Picard et Buot, (p. 5). Deux éclipses favorisèrent les premiers travaux de nos savants, et vers la fin de l'année M. Auzout 
écrivit sur la question des diamètres apparents à M. Oldenbourg, secrétaire de la Société royale d'Angleterre. * C'est ainsi que l'Académie qui se formoit à Paris entroit déjà en commerce de découvertes avec les Académies étrangères. Rien ne peut être plus utile que cette communication, non seulement parce que les esprits ont besoin de s'enrichir des vuës les uns des autres, mais encore parce que différens Païs ont différentes commodités et différens avantages pour les Sciences. La Nature se montre diversement aux divers habitans du Monde; elle fournit aux uns des sujets de réflexion qui manquent aux autres, elle se déclare quelquefois plus ou moins, selon les lieux en enfin pour la découvrir, il n'y a point trop de tout ce qui peut nous être connu (pp. 8-9). ,

Aux sept mathématiciens qui formaient le premier noyau de l'Académie, le roi demanda bientôt à Colbert de joindre des physiciens : de la Chambre et Perrault, médecins, Du Clos et Bourdelin, chimistes, Pecquet et Gayant, anatomistes, Marchand, botaniste. On leur adjoignit des jeunes gens * propres à les aider dans leurs travaux, et à leur succéder un jour ": Niquet, Couplet, Richer, Pivert, de la Voye. Et l'on choisit pour être secrétaire de cette Académie J.-B. du Hamel, prêtre dont la vaste érudition lui permettait d'entendre les différents travaux de ses collègues. Enfin, lc 22 décembre 1666, l'assemblée, dûment constituée, peut-clle se réanir pour la première fois à la Bibliothèque du roi. C'est donc à cette datè du 22 décembre 1666, qu'on peut fixer très exactement la naissance de l'Académie royale des Sciences.

Cette Académie a travaillé au début, comme elle a pu, avec des moyens limités; mais on s'est employé très vite à lui installer de beaux cabinets de travail, pour ne pas employer le mot trop moderne de laboratoires, et à lui fournir les instruments dont elle avait besoin. On construisit l'Obscrvatoire sur les plans de Perrault et d'après les instructions de Cassini au point de vue technique astronomique. On perfectionna le Jardin des Plantes. Et le travail sćricux put se développer dans les meilleures conditions, surtout lorsque l'Académie reçut un règlement précis, strict, qui ne lui avait pas été donné en même temps que ses lettres patentes. Un règlement est indispensable dans toute assemblée de savants pour éviter que les discussions ne tournent en conversations particulières ou en débats violents, inutiles et peu sérieux. Aussi, en 1699, le roi décida qu'il fallait donner un règlement très détaillé, aux membres de son Académie royale des Sciences. C'est là, 
en quelque sorte la seconde naissance de l'Académie royale des Sciences : elle date du 26 janvier 1699. Nous fêtons cette annće le $250^{\circ}$ anniversaire; mais $M$. Gauja, secrétaire archiviste de l'Académie des Sciences, a fait ici méme, il y a peu de temps, une conférence à ce sujet, et je ne m'y étendrai pas 4 .

Le règlement de 1699 prévoyait que, désormais, tout ce qui avait été dit dans l'Académie devait constituer des mémoires, être consigné sur des registres, et que tous les ans, ce qui paraitrait le plus intéressant serait publié en volume.

Fontenclle, qui avait été nommé secrétaire de l'Académie, en 1697 , fut donc chargé, en 1699 , de cette publication et fit précéder chaque recucil de Mémoires d'une Histoire qui leur sert dintroduction où il résume les plus importants, les rend claires et compréhensibles pour tous. Le premier volume, de 1699, parut en 1702; il comporte une préface sur \& L'utilité des mathématiques et de la physique, et sur les travaux de l'Académie des Sciences $>$.

Je ne vous parlerai pas en détail de cette préface sur l'utilité des mathématiques, car vous savez tous ce qu'on peut dire sur l'utilité des mathématiques et de la physique. Ce qui me paraît beaucoup plus intéressant pour notre propos, dans cette Semaine de Synthèse, c'est de citer ce que Fontenelle dit du ròle de la synthèse ou de l'absence de la synthèse, des systèmes ou de l'absence de systìmes, dans les travaux de l'Académie.

«Amassons toujours des vérités de Mathémathique et de Physique au hasard de ce qui en arrivera, ce n'est pas risquer beaucoup. Il est certain qu'elles seront puisées dans un fonds d'où il en est déjà sorti un grand nombre qui se sont trouvécs utiles. Nous pouvons présumer avec raison que de ce même fonds nous en tirerons, plusieurs, brillantes dès leur naissance, d'une utilité sensible et incontestable. Il y en aura d'aulres qui allendront quelque temps qu'une fine méditation ou un heureux hasard découvre leur usage. Il y en aura qui prises séparément seront stériles, et ne cesseront de l'être que quand on s'avisera de les rapprocher. Enfin au pis aller, il y en aura qui seront éternellement inutiles s. Mais, peu importe que les vérités accumulées ne servent pas, l'essentiel

4. Voir Revue d'histoire des Sciences, t. II, numéro sept-déc. 1949. pp. 293-310.

5. Histoire de l'Académie rogale des Sciences, année M.DC.XCIX, Paris, chez Jean Bourdot, 1702, p. 11. 
est la démarche même del l'esprit qui les accumule, et Fontenelle ajoute aussitôt : \& Il est toujours utile de penser juste, mème sur des choses inutiles.

Enfin, voici un des textes tout à fait capitaux de cette préface : * Nous sommes obligés à ne regarder présentement les Sciences que comme ćtant au berceau, du moins la Physique. Aussi l'Académie n'en est-elle encore qu'à faire une ample provision d'observations et de faits bien avérés, qui pourront être un jour les fondements d'un Système, car il faut que la Physique systématique attende à élever des Edifices que la Physique expérimentale soit en état de lui fournir les matériaux nécessaires (p. XVIII). Nous voyons là un Fontenclle prudent, ennemi des systèmes précipitćs, inspiré plus par la méthode cartésienne que par le système clos des Principes. D’ailleurs, il poursuit : * Jusqu'à présent l'Académie des Sciences ne prend la Nature que par petites parcelles. Nul systime général, de peur de tomber dans l'inconvénient des systèmes précipités, dont l'impatience de l'esprit humain ne s'accommode que trop bien, et qui, étant une fois ćtablis, s'opposent aux vérités qui surviennent. Aujourd'hui on s'assure d'un fait, demain d'un autre qui n'y a nul rapport. On ne laisse pas de hasarder des conjectures sur les causes, mais ce sont des conjectures.

«... Le temps viendra peut-être que l'on joindra en un corps régulier ces membres épars, et s'ils sont tels qu'on les souhaite, ils s'assembleront en quelque sorte d'eux-mèmes. l'lusicurs vérités séparées, dès qu'elles sont en assez grand nombre, offrent si vivement à l'esprit leurs rapports, et leur mutuelle dépendance, qu'il semble qu'après avoir été détachées par une espèce de violence les uns d'avec les autres, elles cherchent naturellement à se réunir (p. XIX). , ll me semble voir là trís nettement les deux mouvements de l'esprit : d'une part avec l'accumulation des faits, l'analyse, d'autre part, avec la combinaison des vérités séparées qui s'assemblent selon leurs rapports, la synthèse.

Pour Fontenelle, à ce moment-là, c'est-à-dire en 1700, nous n'en sommes encore qu'à l'époque des analyses : il faut faire des analyses très soigneuses, en attendant de faire des synthèses qui ne soient pas seulement des rêveries métaphysiques. C'est là le but que Fontenelle, dans cette Préface, assigne aux travaux de l'Académie.

Mais il nous faut bien constater que les Académiciens sont loin d'avoir toujours respecté cette règle de sagesse. Parcou- 
rons rapidement les Eloges des savants que Fontenelle a prononcés comme secrétaire perpétuel de l'Académie. Nous verrons que l'esprit de système, en dépit des belles assurances par Iesquelles Fontenelle ouvre la Collection des Mémoires de l'Académie, était tout de même très en honneur. Même avant 1699, dans l'ancienne Académie, celle de 1666, cet esprit de système sc manifestait tout au moins parmi certains académiciens, sinon dans le corps tout entier de l'Académie, chez les partisans de ce que l'on appelait « l'ancienne géométrie * et les partisans de la « nouvelle géométrie > : l'on se battait trés fort entre les deux clans. La « nouvelle géométrie » était celle de Descartes : l'Académie, dans ses débuts, n'était pas encore entièrement cartésienne. Elle compte des savants, ennemis des nouveautés, qui veulent maintenir les méthodes des Anciens en matière de Géométrie. Ainsi Viviani, l'abbé Gallois, Ozanam. Mais le plus acharché à défendre les Anciens est Rolle, qui, d'humeur batailleuse, ne manque pas une occasion d'attaquer ses confrères partisans des Modernes. En 1710, encore, dans l'Histoire de l'Académie royale des Sciences, on peut trouver des échos de ces disputes entre Rolle et La Hire, Rolle disant que tous les problèmes peuvent être résolus avec les anciennes méthodes, La Hire ayant pris parti pour Descartes et montrant qu'il fallait savoir l'interpréter. Fontenelle se fait volontiers l'écho de ces discussions, car n'est-ce pas pour lui un reflet de ses pensées sur la Querelle des Anciens et des Modernes?

Une fois la géométrie de Descartes bien installée au sein de l'Académie, les idées nouvelles, introduites par Lcibniz et Newton, amènent d'autres discussions pour ou contre le calcul infinitésimal. Le calcul infinitésimal a ses détracteurs très violents; évidemment, ce sont à peu près les mêmes que les partisans de la géométrie des Anciens; Rolle toujours, Gallois avec lui, qui discutent de façon assez peu courtoise avec Varignon et tout particulièrement avec Saurin. Même des savants aussi célèbres que Tchirnhans admettent très bien le nouveau calcul, mais ne veulent pas l'employer. Il y a là des résistances aux nouveautés qui sont très manifestes et une grande partie de l'Académie apparaît comme assez conservatrice. D'ailleurs peut-être est-ce un bien, en un certain sens : ne faut-il pas qu'un académicien conserve un cadre de tradition? Cependant l'Académie finit par rétablir la « paix des infiniment petits s en reconnaissant qu'il y avait bien encore des difficultés à éclaircir dans le nouveau calcul, mais 
que vouloir renverser le système total était une offense aux oreilles savantes.

Cependant une autre discussion très vive va se poursuivre au sein de l'Académie, et pendant tout le cours du xvili siècle : c'est la lutte entre deux — je ne sais pas si je dois dire * systèmes > ou * synthèses > : je crois que je dirais plutôt « systèmes * : entre d'une part le cartésianisme pris dans le sens étroit de système de physique et non pas dans celui de la méthode cartésienne, et d'autre part, le newtonianisme et toutes les idées qu'il apporte; c'est la lutte entre les partisans de Descartes et ceux de Newton, entre les partisans de l'impulsion et ceux de l'attraction. Mais je n'en veux rien dire! Je ne parlerai pas de la théorie newtonienne : je veux laisser ce soin à $M$. Koyré qui a bien voulu assister à cette séance et qui, mieux que personne, peut nous exposer ce qu'a été la synthèse newtonienne. Je veux simplement citer un texte de Fontenelle tiré de l'Eloge de Montmor : *Il est certain que si l'on veut entendre ce qu'on dit, il n'y a que des Impulsions; et si on ne se soucie pas de l'entendre, il $y$ a des Attractions et tout ce qu'on voudra; mais alors la Nature nous est si compréhensible, qu'il est peut-être plus sage de la laisser là pour ce qu'elle est ${ }^{6}$. Quelle différence entre ce Fontenelle qui nous disait qu'il ne fallait pas faire de système, que l'Académie allait apporter le plus possible de faits contrôlables et se garderait des généralisations trop hâtives. Ici, il montre combien il est imperméable à ce système de Newton; et je ne crois pas qu'il parle seulement en son nom personnel : il exprime en tant que secrétaire perpétuel l'avis même de l'Académie royale des Sciences.

Un autre texte est encore plus net : dans l'Eloge de Saurin, Fontenelle parlant de son Traité sur la Pesanteur écrit : * Au milieu des difficultés dont il se sent environné il paroit toujours bien convaincu que les vrais Philosophes doivent faire tous leurs efforts pour conserver les Tourbillons de Descartes, sans quoi, dit-il, on se trouveroit replongé dans les anciennes ténèbres du Péripatétisme, dont le Ciel veuille nous préserver. On entend assez qu'il parle des attractions newtoniennes ?. \& Et avec quel enthousiasme Fontenelle donne-t-il son accord aux paroles de Saurin! * Eût-on cru

6. Eloge de Montmort, in Histoire de l'Académie ropale des Sciences, année M.DC.XIX, Paris. Imprimerie Royale, 1, 1721, Histoire p. 91.

7. Eloge de Saurin, in Histoire de l'académie royale des Sciences, année M.DC.XXXVII, Paris, Imprimerie Royale, 1740, Histoire pp. 116-117. 
qu'on dût jamais prier le Ciel de préserver les Français d'un système incompréhensible! Eux qui aiment tant la clarté! Et d'un système né dans un ciel étranger, eux qui aiment tant ne goûter que ce qui leur appartient! , Comment douter d'après ces textes que l'Académie ait été, tout au moins dans cette période, conservatrice?

J'en vois la preuve également dans les paroles d'un secrétaire perpétuel de l'Académie, de beaucoup postérieur, Condorcet, qui a écrit les éloges des académiciens les plus remarquables morts de 1666 à 1699 , éloges que ni Du Hamel ni Fontenelle n'avaient prononcés. Condorcet a très bien remarqué cet esprit de système au sein de l'Académie : * L'ancienne Académie s'est fort occupée dans ses premières années de la cause de la pesanteur... Presque tous nos physiciens se livrèrent alors à des conjectures et à des hypothèses, lorsqu'il eût fallu observer et calculer. Aussi, tandis qu'ils se repaissaient encore de systèmes vagues et d'une vaine métaphysique, Newton avait découvert les lois du systèmes du monde ${ }^{8}$.

On pourrait citer d'autres textes intéressants : ainsi quand Condorcet reproche à Claude Perrault dans certains morceaux de physique, « une teinte de cet esprit systématique quon confondait alors avec l'esprit philosophique $>{ }^{9}$, et aussi dans lEloge de Duclos : « Cette fureur de se croire obligé de rendre raison bien ou mal de tous les phénomènes était la scule chose qui était commune à la philosophie de Descartes et à celle de l'Ecole; elle s'était même encore accrue chez les Cartésiens, dont les explications, plus plausibles et plus piquantes par leur nouveauté et par leur clarté apparente, ctaient appuyées sur l'idée sublime de soumettre aux lois micaniques du mouvement tous. les phénomènes de la nature ${ }^{10}$. $\gg$ De tous ces textes on peut conclure que l'Académie était très conservatrice, très peu accueillante aux idées de l'extérieur, plus attachée à une doctrine qu'à l'examen impartial des faits. Et si regrettable que cela nous paraisse, c'est dans les propos de Maupertuis à l'Académie de Berlin, quil nous faut aller chercher les véritables textes qui nous semblent être la charte de ce que $M$. Le Lionnais appelait hier le rationalisme expérimental.

8. Eloge de Frémile, in Eloges des académiciens de l'Académie royale des Sciences morts depuis 1666, jusqu'en 1699. Paris, 1773, p. 35.

9. Eloge de Duclos, id., p. 67.

10. Floge de Perrault, id., p. 98. 
J'ai passé sous silence toute l'histoire de cette Académie de Berlin, et je n'ai point parlé non plus de ces académies de provinces nées de sociétés réunies chez des particuliers, comme nous en avons vu à Paris ou créées par lettres patentes du roi comme celle de Montpellier qui avait des rapports très étroits avec l'Académie royale de Paris : tous les ans elle devait envoyer un rapport à l'Académie de Paris et entretenir avec elle une correspondance régulière. Il serait beaucoup trop long de relater l'histoire de ces Sociétés provinciales. Je me contenterai de revenir à l'Académie de Berlin, créé par lettres patentes de Frédéric II, en 1700, qui donna d'abord ses mémoires en latin, puis qui les publia en français, lorsque Maupertuis, appelé par Frédéric II, y prit une place prépondérante.

C'est dans les textes de Maupertuis qu'on trouve ce que devrait être, me semble-t-il, et ce qu'a été en fait, la véritable science expérimentale, la science newtonienne.

Alors quil est président de l'Académie de Berlin, Maupertuis prononce un discours sur les devoirs de l'Académicien : dans les problèmes physico-mathématiques que le Géomètre résout, il doit « se ressouvenir toujours des abstractions qu'il a faites : que ses solutions ne sont justes qu'autant qu'il n'y aurait dans les corps que ce petit nombre de propriétés qu'il considère : et que conme il n'y a peut-être point dans la Nature de corps qui soient réduits à ces seules propriétés, il doit, sur ceux qui ont été les objets de ses calculs, consulter encore l'expérience, pour découvrir si des propriétés dont il a fait abstraction, ou dont il a ignoré la présence, n'altèrent pas les effets de celles qu'il a conservées ${ }^{11}$. ,

Un peu avant ce texte et dans ce même discours, Maupertuis donnait les règles de l'expérimentation; on pourrait croire lire un texte de Claude Bernard : les idées, en tout cas, sont à peu près les mêmes. "Cette science (il s'agit de la - Philosophie expérimentale s, nom que portait la première classe des académiciens de l'Académie de Berlin) est toute fondée sur l'expérience; sans elle, le raisonnement, toujours exposé à porter à faux, se perd en systèmes qu'elle dément. Cependant l'expérience a besoin aussi du raisonnement; il épargne au Physicien le temps et la peine; il lui fait saisir tout à coup certains rapports qui le dispensent de plusieurs

11. Maupertuis, Euvres, t. III, Berlin et Lyon, 1753, p. 151. 
opérations inutiles, et lui permet de tourner toute son application vers les phénomènes décisifs.

* Que le Physicien s'applique donc à examiner soigneusement les expériences faites par les autres; qu'il n'ait pas plus d'induigence pour les siennes propres; qu'il n'en tire que des conséquences légitimes; et surtout, qu'également éloigné de l'ostentation qui fait produire le Merveilleux, et du Mystere qui tient caché l'Utile, il les expose à ses Confrères avec toutes leurs circonstances.

N'est-ce pas là exactement l'exposé de la méthode expérimentale? Elle consiste en un aller et retour continuel entre la théorie et l'expérience, soit qu'on parte d'un raisonnement et qu'on en tire par le raisonnernent des conséquences qu'on doit vérifier ensuite par les expériences; soit qu'on parte d'expériences dont les résultats suggéreront des rapprochcments, feront naitre des suggestions, serviront de base au raisonnement.

Fontenelle, en 1699, avait donné des règles incomplètes : il recommandait à l'Académie de faire des collections de faits et de laisser pour plus tard les systèmes. Maupertuis va plus loin et donne la théorie de la méthode expérimentale. Fontenelle, à son époque, avait raison, et l'histoire de l'Académie nous a montré que ces simples préceptes n'avaient pas été respectés, et que Fontenelle lui-même était tombé dans le défaut qu'il soulignait, en soutenant le système de la physique cartésienne contre les idées nouvelles de la physique expérimentale. Pourtant il serait injuste à l'égard de Fontcnelle de ne pas noter quil a vu mieux que quiconque le danger des systèmes, même du système de Descartes, et la différence entre sa Méthode et sa doctrinc. II y a à ce propos dans la Digression sur les Anciens et les Modernes, de 1688, un passage très net qu'il faut citer : «Aristote n'a jamais fait un vrai Philosophe, mais il en a beaucoup étouffé qui le fussent devenus s’il eût été permis. Et le mal est qu'une fantaisie de cette espece une fois établic parmi les Hommes, en voilà pour longtemps : on scra des siccles entiers à en revenir, même après quon en aura reconnu le ridicule. Si lon allait s'entêter un jour de Descartes et le mettre à la place d'Aristote. ce seroit à peu près le même inconvénient 12. » Ne voit-on pas ici, clairement Fontenelle qui, d'autre part, prend fait et cause pour défendre la physiquc

12. Fontenelle, Euvres, Paris, Brunet, 1758, t. IV, p. 199. 
cartésienne, pressentir nettement le danger qu'il y aurait à maintenir, contre la raison même, l'intégrité du système cartésien?

Dans la même Digression, il insiste encore plus : « Avant M. Descartes, on raisonnoit plus commodément; les siècles passés sont bien heureux de n'avoir pas eu cet Homme-là! C'est lui, à ce qu'il me semble, qui a amené cette nouvelle méthode de raisonner beaucoup plus estimable que sa Philosophie même, dont une bonne partie se trouve fausse ou fort incertaine, selon les propres règles qu'il nous a apprises ${ }^{13}$. >

Ce texte me semble capital. J'aurais aimé le citer hier, au moment de la discussion entre M. Cuvillier et M. Le Lionnais sur les différents aspects de l'interprétation de Descartes. Il y a deux choses à voir dans l'œuvre de Descartes : la Méthode et la Doctrine, en particulier les Principes, qui contredisent souvent la Méthode. Je trouve que ce texte est essentiel. On devrait l'avoir toujours présent à la mémoire lorsqu'on veut étudier Descartes. Il éviterait qu'on commette bien des contresens sur la Physique cartésienne.

Mais, revenons à Fontenelle, après avoir été à Berlin en compagnie de Maupertuis. Revenons avec lui, dans ce salon, dans cette demeure qui fut celle de la marquise de Lambert. S'il n'était si tard, il serait très intéressant de parler longuement de ce salon, de ceux qui l'ont suivi, du rôle qu'ils ont joué dans l'histoire des idées au xvir' siecle. Je me contenterai de quelques indications très rapides, m'étant étendue plus sur les Académies dont l'histoire est moins connue.

Le salon de Mme de Lambert ouvert environ en 1690, n'a pris une véritable activité littéraire et scientifique qu'en 1710 . Qu'en dirai-je? Pour être brève et pour employer la méthode suivic jusqu'ici, je citerai des textes de l'époque, écrits d'ailleurs dans une très belle langue.

Du président Hénault : *Voici une maison toute différente des autres : c'est celle de Mme la marquise de Lambert. Elle est connue par quelques pièces de morale qui ont fait estimer son talent pour écrire, la délicatesse de son esprit et sa connaissance du monde. On s'apercevoit qu'elle étoit voisine du temps de l'Hôtel de Rambouillet; elle étoit un peu apprêtée et n'avoit pas eu la force de franchir les barrières du collet monté et du précieux : c’étoit le rendez-vous des hommes célèbres : Fontenelle, Sacy, l'abbé Mongault, etc... Il falloit

13. Id. $I b$., j., pp. 182-183. 
passer par elle pour arriver à l'Académie françoise; on y lisait les ouvrages prêts à paraître. Il y avoit un jour de la semaine où l'on y dînoit, et toute l'après-dînée étoit employée à ces sortes de conférences académiques; mais le soir, la décoration changeoit ainsi que les acteurs. Mme de Lambert donnoit à souper à une compagnie plus galante; elle se plaisoit a recevoir les personnes qui se convenoient; son ton ne chongeoit point pour cela, et elle prêchoit la belle galanterie à des personnes qui alloient un peu au-delà. J'ćtois des deux ateliers : je dogmatisois le matin et je chantois le soir ${ }^{14}$.

Grâce à un article d'Emmanuel de Broglie, extrait du Correspondant, de 1893 , que m'a prêté le général Dumas, je ferai remarquer que le président Hénault dit là une chose fausse : il s'agissait non pas de réceptions qui avaient licu le même jour, mais au contraire de réceptions qui se donnaient à deux jours différents : le mardi et le mercredi, comme lattestent de nombreuses correspondances des contemporains ${ }^{15}$.

Comment mieux résumer le caractère de ce salon que de citer encore Fontenelle? Mme de Lambert \& établit dans Paris une maison où il était honorable d'être reçu. C'était la seule, à un petit nombre d'exceptions près, qui se fût préservie de la maladie épidémique du jeu; la seule où l'on se trouvât pour se parler les uns les autres, et même avec esprit, selon l'occasion ${ }^{16} \gg$. Ne croyez-vous pas que le Centre de Synthise a continué à entretenir cette bonne tradition!

Pour ne pas retarder plus le plaisir que nous aurons d'entendre M. Vernicre, je rappellerai seulement que ce salon, avec celui de la duchesse du Maine, à Sceaux, fut suivi par ceux de Mme de Tencin, de Mme Geoffrin, de Mme d'Epinay, de Mlle de Lespinasse. Le salon de Mlle de Lespinasse prend d'ailleurs un tout autre caractère que les salons précédents, salons littéraires qui avaient grandement hérité des habitudes de l'Hôtel de Rambouillet. Avec Mlle de Lespinasse, avec le baron d'Holbach, avec Mme Necker, nous aurons des salons avant tout politiques, où l'on étudie des questions sociales et

14. Mémoires du Président Hénault, Dentu, 1855, p. 103.

15. * Sur le Salon de Mme de Lambert », voir cet article et surtout une brochure qu'un ami du Centre, un historien, élève de M. Berr, M. Robert Dauvergne, vient de publier chez Albin Michel, a la suite de ses découvertes dans les cartons des Archives.

16. Floge de Mme la marquise de Lambert, in Fontenelle, Euvres, Paris, Brunet, 1758, t. IX, pp. 384-385. 
économiques, et nous sommes vraiment arrivés dans le milieu de l'Encyclopédie, dont va nous parler M. Vernière. Nous entrons tout à fait en contact avec ce monde des Encyclopédistes, nous voyons s'épanouir l'esprit de l'Encyclopédie dont les premiers traits avaient paru ici-mème, dans le salon de Mme de Lambert.

Pour terminer, laissez-moi citer encore un texte, le dernier, cette fois, une lettre de Diderot, où il montre l'esprit de ces derniers salons, et de la société du milieu du xvir' siècle. * Chaque siècle a son esprit qui le caractérise. L'esprit du nôtre semble être celui de la liberté. La première attaque contre la superstition a été violente, sans mesure. Une fois que les hommes ont osć d'une manière quelconque donner l'assaut à la barrière de la religion, cette barrière, la plus formidable qui existe, comme la plus respectée, il est impossible de s'arrêter. Dès qu'ils ont tourné des regards menaçants contre la majesté du ciel, ils ne manqueront pas, le moment après, de les diriger contre la souveraineté de la terre. Le câble qui tient et comprime l'humanité est formé de deux cordes : l'une ne peut céder sans que l'autre vienne à rompre ${ }^{17}$. $>$

C'est de cela sans doute que $M$. Vernière va nous parler maintenant.

M. Berr. - Vous devinez d'après ce que vous venez d'entendre combien me sont précieux le concours, la collaboration, qui datent d'un certain nombre d'années, de ma chère Suzanne Delorme. Vous avez pu juger comme elle connait ce $\mathrm{xvIH}^{\circ}$ siècle, comme elle est renseignée sur la naissance des académies. Elle nous a donné là un fruit précieux de ses études, de ses recherches, et nous attendrons avec impatience sa thèse sur Fontenelle dont nous pressentons la valeur.

Dans chacune de nos étapes nous avons montré qu'il y avaif à la base un mouvement, un épanouissement de curiosité scientifique, au bout desquels se produisait un essai, ou des essais, de synthèse.

Je n'ajouterai à ce que nous a dit Mlle Delorme qu'une chose, en remontant un peu plus haut : il faudrait tout de même citer Peiresc.

17. Lettre de Diderot à la princesse Dashkoff, 3 avril 1771. 
Mlle Delorme. - Je l'avais noté dans mes papiers : je devais en parler en rapprochant son rôle de celui de Mersenne. C'est en effet une lacune.

M. Berr. - Je ne considère pas cela comme une lacune. Mais celui qu'on a appelé le * procureur général de la République des lettres a été un grand excitateur; il a été en rapports avec tous les savants de son époque, il en a reçu un grand nombre. Je ne reproche pas du tout à Mlle Delorme de ne pas l'avoir dit, car c'est un gros chapitre de l'histoire des sciences. Peiresc et ses amis, à commencer par Gassendi, écrivaient en latin: le latin a servi dans cette période de moyen de communication commode et précieux entre les savants de l'Europe entière; mais ce latin les a desservis dans la suite des temps.

Un petit détail personnel et amusant : à l'époque lointaine où je voulais faire ma thèse française sur Gassendi, où j'étudiais tout ce milieu, j'avais l'idée de donner à mon livre ce titre : Le Pays latin, car j'avais trouvé cette expression " le pays latin » à différentes reprises dans des lettres de gens de cette époque. Alors quelqu'un, une autorité universitaire, m'a dit : «On croira que vous écrivez une thèse sur le «Quartier latin ». Quelle horreur! *

A propos des ressources matérielles qui ont toujours ćté nécessaires, et qui souvent ont manqué, et qui manquent encore trop souvent, pour le progrès de la science, je voudrais rappeler que Descartes Iui-même a dit quelque part, dans un texte que je n'ai pas sous les yeux, que, pour permettre les expériences utiles, pour assurer l'avenir de la science et de la pratique, beaucoup d'argent serạit nécessaire, et qu'il fallait espérer que les rois et les particuliers...

Mlle Delorme. - C'est dans la sixième partie du Discours de la Méthode.

M. Bern. - Bref, il a dit que la question dargent était capitale. Il a réclamé par anticipation une caisse de la recherche scientifique. Il a prévu beaucoup de choses!

M. Koyré, j'espère que vous pourrez nous rester, M. Vernière a un sujet important à traiter. Il est déjà 5 heures : si nous commencions maintenant à parler de Newton et à discuter sur lui, je crois que cela nous mènerait bien loin!

M. Vernière est venu de Bordeaux pour nous parler de l'Encyclopédie, de Diderot et de d'Alembert. Il étudie le xvir' siècle passionnément; il fait une thèse que je me réjouis, pour ma part, de lire. Je lui donne la parole. 
M. Vernière. - - Je vous remercie, monsielir, dun aussi encourageant accueil.

Il fut de bon ton, depuis ce qu'on a appelé l'échec du scientisme, de dénigrer l'Encylopédie. Non plus comme l'abbé Barruel pour y voir le grand complot des lumières et la méditation consciente d'une révolution sanglante, mais pour n'y rencontrer qu'une science périmée et une philosophie de seconde main. Au mépris des philosophes se joignait le dédain des historiens de la littérature ou des esthétieiens. Je nıe plais à reconnaître dans l'exposition de 1932, organisée par le Centre de Synthèse et consacrée à l'Encyclopédie et aux encyclopédistes, Ic sionne d'une heurcuse réaction. Depuis cette date, le renouvellement des études sur Diderot, la publication de sa Correspondance, les ouvrages de Jean Pommier et Franco Venturi sur sa jeunesse, de Jean Thomas, Luppol, et Luc sur sa philosophie, la magistrale biographie d'André Billy, ont jalonné ce renouveau d'intérêt. En France, Louis-Philippe May a éclairé maintes sources de l'Encyclopédie, en révélant dans la Revue de Synthèse les registres des éditeurs (1938). Franco Venturi, en 1946, a sondé les origines de l'ouvrage. En Amérique de nouvelles équipes se passionnent pour le contenu même : Alfred Richard Oliver étudie la doctrine musicale des encyclopédistes (Columbia, 1947); Joseph Barker, Diderot et l'Encyclopédie face au christianisme (1941); Gordon et Norman Torrey retrouvent les bonnes feuilles des dix derniers volumes et permettent de juger la censure que leur fit subir, aux grands cris de Diderot, l'imprimeur Le Breton (Columbia, 1947).

Mais si l'histoire même de l'Encyclopédie ne semble plus recéler de mystères importants, il n'en va pas de même de son interprétation et de son sens. Il demeure extrêmement difficile à un travailleur isolé d'extraire de l'Encyclopédie une doctrine cohérente. Dix-sept tomes in-folio sont un obstacle respectable et les recherches de $M$. René Hubert, si précicuses qu'elles soient, n'engagent qu'un domaine restreint, celui des sciences sociales : toutes ses conclusions ne sont pas valables pour d'autres domaines, précisément les plus dangereux. Je n'ai pas la prétention de modifier cette interprétation, mais je crois qu'il n'est pas inutile, lorsqu'il s'agit, dans l'ondoyant royaume de l'histoire des idées, de donner sa place équitable 
à l'Encyclopédie, de préciser à quels besoins l'ouvrage répondait, quels desseins se fixaient ses promoteurs, enfin dans quelle mesure les réalisations ont répondu aux intentions.

\section{1. - Situations et besoins}

Une époque n'est intellectuellement en équilibre que lorsqu'elle se juge capable de donner une explication du monde et de l'homme. Cet équilibre se contente aisément de systèmes clos et de pensée paresseuse. Mais il répond aussi à un besoin incoercible de l'esprit. A l'aube du xvir' siècle en tout cas, cet équilibre est rompu. L'aventure commence ou recommence : ère de conquête après la stabilité, ère de critique plus que de construction, et par là même d'anarchie plus que d'ordre. Sans vouloir résumer les deux magnifiques ouvrages de Paul Hazard, en nous bornant à l'aspect français et non européen du mouvement des lumières, proposons quelques perspectives qui nous semblent s'imposer.

1. L'éclatement du cartésianisme qui se précisait dès la mort de Descartes (1650) est définitif. D'impressionnants systèmes se sont construits dans son sillage, ont séduit quelques disciples, mais n'ont pas infléchi l'évolution intellectuelle : Mallebranche est traité de poète sublime. Spinoza d'antéchrist, Leibniz de théologien scolastique. A l'aube du xviI' siècle une scission s'est faite dans le cartésianisme. Des Méditations est issue l'apologétique cartésienne, avec Fénelon, François Lamy, Régis : au xvir' siècle, ce sera le cartésianisme des cardinaux avec Gerdil à Turin, Bernis et Polignac en France. Issuc du Traité du Monde, la physique cartésienne se défend à l'Académie des Sciences : ce sera le cartésianisme des académiciens, avec Fontenelle et Dortous de Mairan. Du Traité de l'Homme et du Fotus est issu l'iatro-mécanisme dont Boerhaave, à Leyde, est le plus illustre représentant.

Mais cet celatement ne va pas sans danger : une fois débitée, la doctrine n'a plus de puissance offensive. En 1722 , l'abbí Houtteville sonne le glas des apologies rationnelles avec sa l'érité de la religion chrétienne démontrée par les faits. En 1734, une des Lettres philosophiques de Voltaire révèle le monde de Newton et ridiculise les tourbillons. Descartes est défendu en France par chauvinisme plus que par conviction. Si Maupertuis ne put jamais s'implanter en France, et s'il fut 
réduit à l'ostracisme doré de Berlin, c'est qu'il avait été un des premiers promoteurs de Newton en France. Le pire ennemi de Descartes, l'ordre des jésuites, en est arrivẻ à la tolérance et au respect : c'est un signe des temps; le cartésianisme est maintenant une force de réaction plus que de progrès.

2. Le renouvellement des sciences est une deuxidme perspective. L'analyse cartésienne est dépassée par les mathématiques de l'infini. A l'Académie des Sciences, quelques résistances comme celle de Rolle et de Tschirnhaus sont de courte durée, mais les cartésiens eux-mèmes se rallient vite au calcul des fluxions : Malebranche, le marquis de l'Hôpital, Fontenelle. L'ambitieuse mécanique cartésienne cède la place aux humbles travaux de la physique expérimentale (l'abbé Nollet et les cabinets de Physique). La psychologie, jusquelà domaine des littérateurs et des métaphysiens, devient une science. L'évolution se poursuit depuis la Psychologie des sensations, de Locke jusqu'à la psycho-physiologie que Maupertuis oriente vers la biologie $(1745$, La Vénus physique 1751, Dissertation d'Erlangen) et La Mettrie vers le mécanisme (1745, Traité de l'Ame). Mais surtout l'essor des sciences de la nature est la véritable originalité du siècle : à l'Académie des Sciences, Réaumur poursuit l'effort de l'abbé Bignon. Deux Genevois offrent aux spéculations une voie nouvelle : Bonnet, en découvrant la parthénogenèse des pucerons; Abraham Trembley, en observant la scissiparité des polypes d'eau. Le consul Maillet, dans son Telliamed, soupçonne l'évolution des espèces; Buffon annonce son œuvre.

3. Conséquences : la conséquence est celle d'un désarroi intellectuel croissant. Le mot d'un humble chercheur, Gilles Bazin, ami de Réaumur et bibliothécaire du prince de Rohan, est caractéristique. Devant la découverte de Trembley, il s'exclame : "Un chétif insecte vient de se montrer au monde et change ce que nous avions cru être jusque-là, l'ordre immuable de la Nature (1745). \ Cette inquiétude est partagée par tous, et la réaction normale est un besoin d'ordre inhérent à l'esprit et particulièrement à l'esprit français.

Or, cet ordre ne peut plus être l'ordre chrétien. L'orthodoxie est incapable de donner une explication valable du monde et de l'homme. Cosmologie, ethnologie ne peuvent se fonder sur la Genèse; l'orthodoxie s'épuise à défendre des 
terres contestées : chronologie sacrée du Père Pétau, Pézron, Tournemine; naĩvetés de Dom Calmet; faiblesse de l'apologétique (Houtteville, François, Denyse, Lignac, Pluquet); les découvertes d'Astruc, demeurent ignorées comme les audaces de Richard Simon avaient été condamnées.

Mais cet ordre ne peut être un ordre systématique. Ce qu'on refuse à Dieu, on ne saurait l'accepter de Descartes ou de Leibniz. Vers le milieu du siècle, c'est à qui dénoncera le danger des systèmes: Diderot dans Les Bijoux indiscrets (1747), développe l'allégorie du temple de l'hypothèse et du géant de l'expérience. Le premier Dicours de Buffon en 1749 , et le Traité des Systémes de Condillac la même année sont encore plus violents : on ne veut plus de « châteaux enchantés * (Condillac), d' \& abstractions de notre esprit borné (Buffon), de la manie de l'analogie et de la symétrie.

Cet ordre doit être d'abord un recensement, un inventaire, une Somme. Toute une génération éprouve le besoin de rassembler les connaissances éparses, perdues dans de trop nombreuses publications : Acta Eruditorum, de Leipzig, Philosophical Transactions, de Londres, Journal des Savants, Mémoires académiques, journaux de Hollande. C'est le but de deux grandes cuvres du temps, L'Histoire générale des voyages, commencée en 1746, par l'abbé Prévost, et, depuis 1749, l'Histoire naturelle, de Buffon.

Mais ce classement génétique ou pédagogique est insuffisant. L'éparpillement est un danger. Une idée progresse peu à peu : l'esprit qui découvre les sciences veut découvrir leur lien. L'esprit expérimental, indispensable dans la recherche, est incapable d'organiser. Et voici que beaucoup de bons esprits font retour, non à la métaphysique de Descartes, mais à la méthode cartẻsienne (d'Alcmbert dans l'Excès de critique en matière de religion, La Mettrie dans la préface de ses anures). Bien que le mot ne soit pas prononcé, on sent le besoin d'une synthèse, d'un ordre légitime qui, sans préjuger de l'explication unitaire du monde et de l'explication définitive de l'homme, manifeste du moins l'unité de l'esprit humain, seul ouvrier de la science en formation. Cette synthèse n'est pas un systeme, qui étymologiquement est un ensemble clos, où le réel doit venir tant bien que mal s'inclure, mais qui ne va pas à la recherche du réel. C'est au contraire un mouvement unifiant qui organise et conquiert à la fois, assimile les faits et se laisse non seulement informer mais déformer par eux. 
Or ce besoin de synthèse, ce n'est pas un simple dictionnaire, une vaste compilation qui pouvait y répondre. Entre l'humilité excessive de Locke, son absence d'envol, et les ambitions prématurées de Descartes et de Leibriz, à la recherche l'un d'une mathématique, l'autre d'une caractéristique qui fussent universelles, il $y$ avait un moyen terme, celui d'une encyclopédie. Ce désir d'ordonner et d'unifier sans déformer le monstrueux ensemble de la connaissance était-il légitime? Tous le pensent à mesure que le siècle s'avance. Leibniz en proclamait l'intérêt; Ephraïm Chambers, à Londres, en donne, en 1726, une esquisse dans sa Cyclopodia; le 21 mars 1737, le chevalier de Ramsay demande pour l'exécution de ce vaste dessein l'appui de la franc-maçonnerie européenne : tous devaient s'unir « pour former les matériaux d'un dictionnaire universel des arts libéraux et de toutes les sciences utiles; on $y$ expliquera non seulement le mot technique et son étymologie, mais on donnera encore l'histoire de la science et de l'art, ses grands principes et la manière d'y travailler. De cette façon, on réunira les lumières de toutes les nations dans un seul ouvrage qui sera comme un magasin général de tout ce qu'il y a de beau, de grand, de lumineux, de solide et d'utile dans toutes les sciences naturelles et dans tous les arts nobles. Cet ouvrage augmentera dans chaque siècle, selon l'augmentation des lumières $*$. En 1740, le duc d'Antin, premier grand maître de l'ordre, répète l'appel. A Berlin, le secrétaire de l'Académie, Formey, accumulait déjà des documents qu'il cédera plus tard, devant l'initiative française, aux éditeurs de l'Encyclopédie. Bacon, réédité en 1730 à Londres et à Amsterdam, devient officiellement le père de la philosophie expérimentale dans la douzième Lettre anglaise de Voltaire, avant de patronner le dessein encyclopédique. L'Encyclopédie paraît une nécessité de l'heure, nécessité affirmée plus tard par Voltaire et Malesherbes, et qui révele non pas une mode, mais les exigences intellectuelles d'une génération.

\section{II. - LES DESSEINS DE L’ «ENCYCLOPÉdie *}

Or ces exigences étaient senties par les deux promoteurs de l'Encyclopédie et évoqués dans deux documents essentiels : le Discours préliminaire, de d'Alembert et l'article Encyclopédie, de Diderot. Sans diverger positivement, les deux doctrines se confirment, se complètent, mais engagent aussi 
deux natures d'hommes, ce qui peut expliquer certains heurts dans leur collaboration et certaines failles dans l'œuvre.

1. Le Discours préliminaire. - Ce n'est pas une préface ordinaire, mais un véritable Discours de la méthode (82 pages in-octavo), non seulement par son dessein, mais par son inspiration cartésienne. D’emblée, quelques idées se détachent sur l'ensemble :

A. - Tout d'abord la recherche d'un ordre : l'Encylopidie * doit exposer autant qu'il est possible l'ordre et l'enchainement des connaissances humaines $\gg$, e'est-à-dire qu'elle doit se présenter comme un effort de synthèse et non de compilation. Mais cet ordre doit être prudent : * Les sciences et les arts se prêtent mutuellement des secours. Il y a par conséquent une chaine qui les unit; mais il est difficile de renfermer dans un système qui soit un les branches infinimcint variées de la science humaine. * On croit entendre l'écho de Descartes dans les Regulae : «Les sciences sont tellement liées ensemble qu'il est bien plus facile de les apprendre toutes à la fois que d'en apprendre une seule en la détachant des autres. „ Mais plus méticuleux que Descartes, plus conscient de la complexité du réel, d'Alembert affirme aussitôt le second but de l'Encyclopédie : la recherche, non d'une mithodologie universelle, mais d'une axiomatique propre à chaque science; il faut établir " sur chaque science et sur chaque art soit libéral, soit mécanique des principes généraux qui en sont la base $\gg$. D'Alembert affirme done à la fois une certitude : chaque science a sa méthode, et un espoir, celui de l'unité intellectuelle du monde : «L'univers, pour qui saurait l'embrasser d'un seul point de vue, ne serait qu'un fait unique et une grande vérité. >

B. - Mais que doit être cet ordre? D'Alembert exclut aussitôt l'ordre génétique. La genèse de nos connaissances est anarchique, dépend des hasards et des besoins. Aucune hiérarchie n'apparaît ainsi dans les découvertes : "C'est un labyrinthe, un chemin tortueux où l'esprit s'engage sans trop connaitre la route qu'il doit tenir. $\$$ Il faut au contraire * former un arbre encyclopédique ou généalogique qui rassemble toutes nos connaissances sous un même point de vue et qui serve à en marquer l'origine et le lien s. Cet ordre est un seul ordre philosophique : * L'ordre encyclopédique de nos connaissances consiste à les rassembler dans le plus pelit espace possible et à placer le philosophe au-dessus de ce vaste 
labyrinthe, dans un point de vue fort élevé, d'où il puisse apercevoir à la fois les sciences et les arts principaux, voir d'un coup d'œil les objets de ses spéculations et les opérations qu'il peut faire sur ces objets, distinguer les branches générales des connaissances humaines, les points qui les séparent et qui les unissent, et entrevoir même quelquefois les routes secrètes qui les rapprochent. > En somme le but est de dresser une mappemonde qui ne prétend pas découvrir les moindres accidents de terrains, mais indiquer par des calculs simples et des références exactes la valeur respective des continents, des îles, des montagnes et des fleuves. L'arbre encyclopédique c'est la mappemonde, les articles encyclopédiques seront les cartes particulières et les plans directeurs.

C. - Mais cet ordre doit être prudent. D'Alembert se rallie sans grand enthousiasme à l'arbre baconien de la connaissance, sans se dissimuler son caractère arbitraire. C'est un schéma heureux qui sous trois facultés (mémoire, raison et imagination) range l'histoire, la philosophie et les beauxarts et répond à la distinction banale des érudits, des philosophes et des beaux-esprits. Mais avec quelle prudence ne nous invite-t-il pas à nous en méfier : ce serait « un dénombrement frivole pour qui s'en contenterait $\star$. Rien de plus ridicule qu'un géographe en chambre qui croirait connaitre le monde en consultant un planisphère. Cet ordre, d'Alembert ne nous le cache pas, est avant tout clair, commode, pédagogique.

D. - Enfin cet ordre ne doit pas gêner l'élaboration positive des sciences. L'encyclopédiste doit se garder de toute hypothèse, de toute conjecture aventureuse. Et par là, d'Alembert participe bien au mouvement général de l'empirisme : * Un écrivain qui ferait parmi nous l'éloge des systèmes viendrait trop tard..., si l'esprit de système est quelquefois nécessaire pour nous mettre dans le chemin de la vérité, il est presque toujours incapable de nous y conduire par lui-même. \ Aussi bien la science doit-elle s'enfermer dans les faits observables et dans les conséquences * qu'on en peut déduire $\gg$. L'homme de science ne doit pas s'interroger sur les causes des phénomènes; il peut les entrevoir, * mais c'est au calcul à assurer l'existence de ces causes ».

Le Discours préliminaire nous apparait donc comme un étrange mélange d'enthousiasme et de froideur. Après avoir proclamé comme Descartes l'unité de la connaissance et la 
volonté, non pas de supposer un ordre, mais d'imposer un ordre qui manifeste l'emprise de l'esprit sur la matière, d'Alembert hésite, s'interroge, laisse la prudence développer ses réticences et ses inquiétudes. Mais cette hésitation n'est pas une faiblesse : elle exprime à merveille l'état de la science vers 1749 , cent ans après la mort de Descartes, cent ans avant l'essai de synthèse positiviste. D'Alembert sait qu'il est trop tôt, que l'heure de la synthèse n'est pas venue, qu'il faut encore accumuler et classer les faits, qu'il faut découvrir, organiser avant d'interpréter. Mais son espoir est intact. C'est cette contradiction apparente de l'espoir et de la prudence, ce déchirement intérieur entre l'exigence de la vérité et l'exigence d'un ordre, ce sentiment que la vérité existe mais aussi que la vérité se construit, qui font de d'Alembert un de nos contemporains : la science moderne n'échappe pas encore à ce tourment.

2. L'article «Encyclopédie » de Diderot. - Cette réserve scientifique et cet enthousiasme latent, mais constamment inhibé, ne sont pas le fait de Diderot. L'exercice de la pensée est pour lui un beau risque à courir, une incessante aventure. D'oủ l'intérêt qu'il y a à comparer l'article Encyclopédie avec le Discours préliminaire. 86 pages in-octavo, cet article a l'ampleur sinon la rigide cohérence de la préface de d'Alembert. Mais, d'emblée, nous entrons dans un tout autre monde intellectuel. L'enthousiasme libéré s'exalte avec la foi d'un Rabelais ou d'un Erasme. L'éloquence se fait fervente et pressante. Il ne s'agit plus seulement d'un programme, mais d'un véritable appel à l'opinion.

A. - Une perspective plus qu'un ordre. L'idéc première de Diderot est évidemment que l'Encyclopédie « doit rassembler les connaissances éparses et en exposer le systime général ». Mais ce système (le mot est caractérislique) ne lintéresse guère. Diderot ne dit mot du fameux arbre baconien. L'ordre choisi ne peut être qu'arbitraire : «Il est impossible de bannir l'arbitraire de cette grande distribution première. L'univers ne nous offre que des êtres particuliers, infinis en nombre et sans presque aucune division fixe et déterminée... Tout s'y enchaîne et s'y succède par des nuances insensibles et, à travers cette uniforme immensité d'objets, s'il en paraît qui, comme des pointes de rocher, semblent percer la surface et la dominer, ils ne doivent cette prérogative qu'à des systèmes particuliers, qu'à des conventions 
vagues... et non à l'arrangement physique des êtres.et à l'intention de la Nature.

Ironiquement, Diderot suggère l'existence d'un système divin, d'un plan universel préformé de toute éternité par lintelligence de Dieu. C'est évidemment pour déplorer aussitôt notre ignorance. Aussi n'est-ce pas tant un ordre, eet ordre géométrique plus ou moins postulé par d'Alembert, qu'une perspective neuve qui semble donner son sens à l'Encyclopédie. Dieu n'est plus le centre de cette nouvelle Somme, mais l'homme. Si l'on bannit l'homme, * ce spectacle pathétique et sublime de la Nature n'est plus qu'une scine triste et muette. L'univers se tait. Le silence et la mort s'en emparent. Tout se change en une vaste solitude où les phénomènes inobservés se passent d'une manière inobservée et sourde. C'est la présence de l'homme qui rend l'existence des ètres intéressante. Pourquoi n'introduirions nous pas l'homme dans notre ouvrage comme il est placé dans l'univers? Pourquoi n'en ferions-nous pas un centre commun? L'homme est le terme unique d'où il faut partir et auquel il faut tout ramener ». Page d'un frémissement tout pascalien et qui dépasse infiniment le monde de la science. Diderot ne prétend plus comme Descartes expliquer le monde et l'homme, mais comme Pascal, il s'inquiète de leur sens et de lcur destinćc.

B. - Mais cette perspective ne peut se découvrir qu'à l'aide d'une philosophie de l'histoire. D'Alembert, dans la gencse des connaissances humaines, était frappé par le rôle du hasard. Diderot est au contraire frappé par les coïncidences et le caractère concerté de l'évolution des sciences. C'est avec une espèce d'effroi religieux qu'il perçoit la prodigieuse accélération du progrès : les mots, les opinions, évoluent comme les arts et les sciences. L'Encyclopédie risque de vieillir rapidement si elle n'exprime qu'un état temporaire de la connaissance. Pour éviter ce danger, l'encyclopédiste doit faire ouvre critique, mais aussi œuvre divinatrice : l'Éncyclopédie doit suivre le fil de l'histoire, s'inserire dans le sens de l'évolution.

Pour cela Diderot propose une révision générale des valeurs du passé. Le progrès de la Raison renverse les idoles et les statues, mais « en relève quelques-unes qui étaient renversées ». Une véritable révolution intellectuelle se prépare: - Ce qui donnera à l'ouvrage l'air suranné, c'est surtout la révolution qui se fera dans l'esprit des hommes et dans le 
caractère national, aujourd'hui que la philosophie s'avance à grands pas, qu'elle soumet à son empire tous les objets de son ressort, que son ton est dominant, et qu'on commence à secouer le joug de l'autorité et de l'exemple. \$ Euvre critique donc, mais aussi divinatrice; il ne faut pas seulement connaître, mais devancer son temps : « Connaitre l'esprit de sa nation, en pressentir la pente, le gagner de vitesse, en sorte qu'il ne laisse pas votre travail en arrière, se résoudre à ne travailler que pour les générations suivantes, parce que le moment où nous existons passe, et qu'à peine une grande cntreprise sera-t-elle achevée que la génération présente ne sera plus. Enfin il faut avoir le sens de ce que Diderot appelle les Révolutions, c'est-à-dire des cycles scientifiques, prévoir en quel domaine est la fécondité et quelle science est en stagnation. Le progrès, en efiet, n'est pas linéaire, mais cyclique, comme le voulait Vico que Diderot connaît peutêtre par Boulanger. Certaines sciences comme les mathématiques sont maintenant inertes, nous affirment Buffon et Diderot. C'est l'âge de la biologie qui commence : « Les révolutions sont nécessaires; il y en a toujours eu et il y en aura toujours. Le plus grand intervalle d'une révolution à une autre est donné. Cette seule cause borne l'étendue de nos travaux. Il y a dans les sciences un point au-delà duquel il ne leur est presque pas accordé de passer. \$

C'est ainsi que Diderot pose les jalons d'une véritable philosophie de l'historien; non de l'histoire des peuples : if laisse à Voltaire le soin d'attaquer Bossuet; mais l'histoire de nos conquêtes intellectuelles. L'esprit de Diderot court comme le feu dans la brande, et le programme positif cède souvent à l'imagination, aux prévisions contestables. Rien n'est plus positif que d'exprimer son temps, mais lorsqu'on prélend le devancer, comment résister à la tentation d'agir sur lui ct de transformer une œuvre de science en une œuvre de propagande? Diderot n'a pas reculé devant l'obstacle.

C. -- Aussi bien Diderot ne cache-t-il guère sa recherche de l'efficacité. L'encyclopédiste ne doit pas seulement exposer les résultats de la science, mais proposer des recherches neuves. Le génie peut percevoir des rapports nouveaux entre les sciences. C'est sur ce point que Diderot s'oppose le plus à l'esprit expérimental de d'Alembert; ce dernier interdisait l'hypothìse et la conjecture; Diderot lui répond : « Il vaut encore mieux risquer des conjectures chimériques que d'en 
laisser perdre d'utiles ¿; c'est déjà une revanche de l'intuition.

Mais Diderot visait plus haut. L'Encyclopédie devait agir sur l'esprit même de la nation. De l'aveu même de Diderot. elle comportait * une force interne et une utilité secrète dont les effets sourds seraient nécessairement sensibles avec le temps $\gg$. C'était la raison de ses efforts, l'explication de son enthousiasme et de son acharnement. L'Encyclopédie devait etre la vengeance de Vincennes, qui symbolisait dans sa vie même l'emprise encore dangereuse d'un monde déjà caduc. Or, plus que le pouvoir royal, c'était l'édifice chrétien qui empêchait l'essor des Iumières; Diderot l'avoue crûment et dévoile ses batteries : «outes les fois qu'un préjugé national mériterait du respect, il faudrait l'exposer respectueusement et avec tout son cortège de vraisemblances et de séductions, mais renverser l'édifice de fange, dissiper un vain amas de poussière, en renvoyant aux articles où des principes solides servent de base aux vérités opposées. Cette manière de détromper les hommes opère infailliblement et sans aucune fâcheuse conséquence... Le caractère d'un bon dictionnaire rest de changer la façon commune de penser.

Ce n'est plus Pascal que nous rappelle ici Diderot; c'est plutôt Renan dont nous sentons en cette page à la fois l'onction et l'agressivité secrète, à cette réserve près que la prudence renanienne avait moins d'excuses. L'enthousiasme aussi est plus neuf et plus frais. Cette perspective centrale de l'homme, ce besoin d'ébaucher une philosophie de l'histoire, et de se baigner dans l'immense courant d'une évolution encore obscure, ce désir d'action et d'efficacité, cette espérance de grandir l'homme et de trouver à la fois le langage qui l'entraine et un idéal qui l'exalte, tout cela dépassait singulièrement les desseins limités de d'Alembert. L'Encyctopédie perdait ce caractère anodin qu'elle revêtait dans la dédicace au chancelier d'Aguesseau. Elle ne se bornait pas à préciser les aspirations d'un siècle, elle l'entrainait; avec ferveur, elle appelait l'aventure.

\section{III. - LES RÉlisations DE L'ENCYCLOPÉdie}

Ce double programme a-t-il été réalisé? Honnêtement, nous pouvons dire : non. Car il était en son temps et peut-être encore dans le nôtre irréalisable. Mais l'Encyclopédie sur deux points est une réussite exceptionnellẹ. 
A. - Elle exprime à merveille la civilisation matérielle de son temps, l'état des techniques, des métiers, le détail des costumes, des modes et des goûts. Pour qui a la patience de relire ses articles et de feuilleter ses planches, elle a la même puissance de résurrection que les estampes et les correspondances du temps. Imaginons que nous ayions un tel dictionnaire pour le siècle de Villon ou même pour l'âge de Balzac.

B. - Elle exprime fort bien l'intelligence moyenne de son temps dans ses curiosités les moins dangereuses, histoire, géographie, économie politique, histoire des voyages, ethnologie et sociologie naissantes, histoire des sciences et de la philosophie. M. Hubert a pu constater en ces domaines l'excellence, le nombre de ses sources, et leur correcte interprétation.

Mais l'Encyclopédie n'est-elle que le bilan des connaissances humaines en 1750-1765? N'est-elle qu'une Somme, qu'un inventaire, qu'un recensement hâtif avant la liquidation révolutionnaire? \& Le moment le plus glorieux pour un ouvrage de cette nature, disait Diderot, ce serait celui qui succéderait immédiatement à quelque grande révolution qui aurait suspendu le progrès des sciences, interrompu les travaux des arts et replongé dans les ténèbres une portion de notre hémisphère. \En fait, la Révolution devait venir, sans arrêter l'essor des sciences, mais non sans rendre caduque l'information encyclopédique. Si l'Encyclopédie devait durer, de l'aveu même de ses promoteurs, c'était par son esprit, sa philosophie interne, plus que par son information.

Or rien n'est plus délicat que de retrouver dans l' "Encyclopédie \& l'esprit encyclopédique. Cette rechercice prudente d'un ordre capable d'unifier les sciences, cette décision du philosophe de dominer la connaissance et de dresser la gigantesque mappemonde, cette perspective évolutive, cette lutte audacieuse contre les préjugés, nous avons pcine à les percevoir dans la succession alphabétique des articles, dans les contradictions doctrinales, dans l'érudition mal digérée, dans les précautions grossières à l'égard des pouvoirs, dans les sermons mensongers, dans les dialectiques scandaleuses qu'accumule l'Encyclopédie.

1. L'Encyclopédie est, en effet, une auvre collective, rédigée au dire de Diderot * par des hommes liés par l'intérêt général du genre humain et par un sentiment de bienveillance réciproque . En fait les opinions divergent souvent 
entre les philosophes * tondus \& que sont les abbés Yvon et Mallet, l'athée d'Holbach et le protestant de Jaucourt. Diderot en est souvent dégoûté et refuse dans l'article Editeur d'assumer la responsabilité morale de tous les articles. On pense à la sagesse de Descartes dans les Regulae : «Quand même tous seraient d'accord, leur doctrine ne suffirait pas, si notre esprit n'était lui-même capable de résoudre les problèmes : ce serait avoir appris non des sciences, mais de l'histoire. >

2. Mais l' «Encyclopédie \'est pas libre : c'est une œuvre enchaînée. On la voit dérouler une apologétique orthodoxe dans les articles Adam, Arbre de Vie, Enfer, Christianisme, et une théologie classique dans les articles Providence, Révélation, Théologie. Qui ira chercher les impiétés de l'article Junon? Mêmes contradictions sur les grands problèmes philosophiques : cinq doctrines du droit naturel, selon M. Hubert, le luxe attaqué et défendu (articles Luxe et Scythes), le célibat vanté à l'article Christianisme et honni à l'article Célibat, la liberté morale défendue dans les articles Liberté et Providence et réfutée aux articles Leibnizianisme et Laideur avec une virtuosité de sophiste, les miracles sereinement admis ou ridiculisés; chose plus grave encore, nous voyons la philosophie expérimentale louée dans les articles Philosophie et Préoccupation, le critère empirique attaqué dans l'article Philosophie des Romains, l'esprit de conjecture vanté dans l'article Génie. Nous comprenons dès lors la gène d'un disciple comme Naigeon, qui interprète les déficiences de l'Encyclopédie comme des prudences nécessaires. Il est facile, trop facile de jouer des thèmes alternés de l'ésotérisme et de l'exotérisme. Comment ne pas partager souvent l'irritation de Ducros qui, dans cette incessante « escobarderie », voit « une atteinte a la conscience publique », que Diderot risque d'égarer et non d'éclairer. Les conséquences en sont si graves que nous nous y trompons toujours : Rosenkranz, frappé par la vigueur du ton de l'article Liberté prend Diderot pour un philosophe de la contingence; André Gide, devant l'article Spinoza déclare doctement qu'il n'est * pas si facilo d'être athée $\gg$. Diderot a donc menti sciemment, alors que Descartes, plus honnêtement sinon plus courageusement, renonçait à parler.

3. Enfin, les promoteurs eux-mêmes de $l$ "Encyclopédie ne sont pas sûrs de leur méthode. D'Alembert prône l'empi- 
risme, alors qu'il n'a pas le goût de l'observation, et dédaigne souverainement les faits historiques; demeuré mathématicien, il irrite par sa raideur l'enthousiaste Diderot. D'Alembert, plus cartésien qu'il ne l'avoue, recherche derrière les principes généraux de chaque science une axiomatique universelle. Dans ses Eléments de Philosophie il loue cette réduction dr toute science à un jeu mécanique de signes algébriques et prélude aux ambitions de la science pré-einsteinienne. Plus tard, appliquant le calcul des probabilités à la guérison de la variole, il ouvre le champ d'une science statistique et d'un déterminisme nouveau. Aussi bien, ne saurait-il courir le risque où l'entraine une cuvre de propagande et de vulgarisation : c'est avec soulagement qu'il quitte l'Encyclopédie en 1758.

Diderot lui-même ne cesse de s'interroger; il ne croit pas à la fécondité des mathématiques. L'avenir semble sourire aux sciences de la vie, mais l'empirisme à courte vue d'un Réaumur l'écœure. Tous les ouvrages où sa pensée personnelle se découvre, sa Correspondance, ses notes de travail, ses rêveries, révèlent un vaste besoin d'explication unitaire du monde, et cela en transcendant l'expérience. Le philosophe, aussi bien dans les Pensées Philosophiques que dans les P'nsées sur l'interprétation de la nature, a pour rôle de devancer génialement la science et de lui ouvrir les voies : un regard d'aigle doit compenser la myopie de l'observateur. Et voilii Diderot rêvant à la molécule organique, à la chaine des êtres. à l'évolution des espèces, au gluten qu'on pourrait transformer en protoplasme. Dans ce rêve d'ordre et d'unité, il retrouve le cadre grandiose de l'Ethique auquel les sciences de la Nature pourraient fournir une matiere vivante, une pulpe enrichie. Aussi dès 1758 l'Encyclopédie n'est-elle plus pour Diderot le grand dessein. Il la poursuit par orgueil, par sens de l'honneur plus que par foi et conviction. Quel soulagement en 1765 quand il est libéré de ce bagne! Lorsqu'il insulte Le Breton, coupable d'avoir censuré quelques articles, il y a en lui plus de mauvaise conscience que d'authentique rancœur : il s'itait déjà censuré lui-même.

Faudrait-il conclure à l'inefficacité et à l'inutilité de liEncyclopédie et ne voir en elle qu'une inestimable collection de documents sur la civilisation du temps? Faudrait-il y voir le dernier effort intellectuel, maladroit et incohérent, d'une société qui se meurt? Faut-il être aussi sévère qu'Auguste Comte devant ce monument d'un âge encore métaphysique? 
Nous ne le croyons pas. Malgré ses contradictions, ses prudences et ses roueries, l'Encyclopédie représente une étape nécessaire entre Descartes et l'âge positif; et l'on se demande si le hasard seul la place à égale distance entre la mort de Descartes en 1650 et l'Avenir de la Science écrit en 1849 par Renan.

1. Comme l'esprit cartésien lui-même, l'esprit encyclopédiste représente une tentative de coordination du savoir positif, un effort pour libérer la science de ses entraves théologiques, du prestige de l'autorité et de la tradition. Et si Descartes lui-même en souffre, c'est au nom même de l'esprit cartésien.

2. Comme l'esprit cartésien lui-même, l'esprit encyclopédiste assigne une perspective humaine à l'édification de la science. Diderot a proclamé son but : «a science de l'homme en général > dans laquelle toute science vient s'inclure. Comme Descartes, Diderot ne croit pas que le monde ait un sens en dehors de l'esprit qui le comprend, le construit et l'organise; c'est cette ignorance de l'homme qu'il reprochera à Helvétius, comme Descartes dans son apostrophe fameuse à Gassendi lui reprochait de n'être que chair « $O$ caro *

3. Comme Descartes enfin, l'esprit encyclopédique proclame lunité de la science. Descartes disait : " Il doit y avoir une science qui explique tout ce qu'on peut chercher touchant l'ordre et la mesure, les mathématiques universelles. \ Cet ordre et cette mesure, Diderot et d'Alembert les ont passionnément souhaités, sinon découverts.

4. Mais au contraire de Descartes, l'esprit encyclopédique ne croit pas à l'achèvement de la science. Descartes est pour eux un audacieux Prométhée enchainé par l'orgueil. Le but de Diderot est de donner une synthèse provisoire, non un système du monde. Dans l'article Philosophie il * laisse à la postérité le soin de faire un système complet , et constate que «ce temps est bien éloigné, si tant est qu'il arrive jamais \$. A Lasnière qui écrivait en 1771 un Système général du monde, il répond que * les lumières ne sont pas proportionnées à la tentative $>$.

Aussi la fécondité de l'Encyclopédie n'est-elle pas dans la science qu'elle essaie maladroitement d'organiser, mais dans son dessein, dans son esprit, dans son exemple. Presque tout est caduc dans sa matière, tout est encore vivant dans son 
aspiration. La synthèse provisoire qu'elle réalise est ruinée, la synthèse qu'elle appelle est toujours à faire. Non sculement par l'acte de foi de ses promoteurs, mais par l'échec même de leurs projets, l'Encyclopédie manifeste bien la puissance et la fragilité des liens qui unissent l'homme à l'univers : nous vivons toujours la même aventure, chanceuse et féconde. 